\title{
GERENCIAMENTO DO CUIDADO: ações dos enfermeiros em um serviço hospitalar de emergênciaa
}

\author{
José L uís G uedes dos SA N T OS ${ }^{b}$ M aria Alice Dias da Silva LI M Ac
}

\section{RESUMO}

E studo com objetivo de anal isar as ações de gerenciamento do cuidado real izadas pel os enfer meiros em um serviço hospitalar de emergência. Pesquisa qualitativa do tipo estudo de caso, realizada com enfermeiros do Serviço de E mergência de um H ospital U niver sitário da região sul do Brasil. Os dados for am col etados por meio de observação par ticipante e entrevista semi-estruturada e anal isados pela técnica de análise temática. O s resultados evidenciam ações dos enfermeiros no planejamento do cuidado, previsão e provisão de recursos, supervisão, liderança e capacitação da equipe de enfermagem. No planejamento do cuidado, identifica-se a execução do processo de enfer magem e o controle da realização dos exames labor atoriais e radiológicos. As ações de previsão e provisão de recursos foram: el aboração de escala mensal de funcionários, distribuição diária dos funcionários e gerenciamento de recur sos materiais. A liderança favorece o planejamento da assistência, a coordenação da equipe de enfer magem e a del egação de atividades.

D escritores: G erência. Enfermagem em emergência. Papel do profissional de enfer magem. Serviço hospitalar de emergência.

\section{RESUMEN}

E steestudio tuvo como objetivo analizar la actuación del enfer mero en el gerenciamiento del cuidado en un servicio hospitalario de emergencia. Investigación cual itativa de tipo estudio de caso realizada con los enfermeros del Servicio de E mergencia de un H ospital U niversitario de la R egión Sur de B rasil. L os datos fuer on recogidos a través de observación participante y entrevistas semi-estructuradas y analizados utilizando el análisis temático. L os resultados demostraron las acciones de los enfermeros en la planificación del cuidado, la previsión y provisión de recursos, supervisión, liderazgo y capacitación del equipo de enfermería. E n la planificación del cuidado, se ha identificado la ejecución del proceso de enfermería y control de la realización de los exámenes de laboratorio y radiológicos. L as acciones de previsión y provisión de recursos fueron: escala mensual del trabajo y distribución de los profesionales deenfer mería y recursos materiales. El liderazgo favorecela planificación del cuidado, coordinación del equipo de enfermería y delegación de las actividades.

Descriptores: $\mathrm{G}$ erencia. E nfermería de urgencia. R ol de la enfermera. Servicio de urgencia en hospital. Título: $G$ erenciamiento del cuidado: acciones de los enfermer os en un servicio hospitalario de emergencia.

\section{ABST RACT}

T his study aimed to analyz ecare management actions performed by nurses in a hospital emergency service. This is a qualiative research of the case study type, carried out with nurses from the E mergency Service of a U niversity $\mathrm{H}$ ospital in southern B razil. T hedata were collected through participant observation and semi-structured interviews, and analyzed using thematic analysis. T he results show nurses' actions in care planning, forecasting and provisioning of resources, supervision, leadership and training of the nursing team. In care planning, there is the execution of the nursing process and the control of the realization of laboratory and radiological tests. The actions of forecasting and provisioning of resources w ere: elaboration of the monthly schedule of employees, daily distribution of the staff and the management of material resources. L eadership encourages the planning of care, the coordination of the nursing staff and the delegation of activities.

D escriptors: M anagement. E mergency nursing. N urse's role. E mergency service, hospital.

T itle: Care management: nurses' actions in a hospital emergency service

${ }^{a}$ A rtigo extraído da dissertação de M estrado apresentada em 2010 à E scola de Enfermagem da U niversidade Federal do Rio Grande do Sul.

b M estre em Enfermagem, D outorando pelo Programa de Pós-Graduação em Enfermagem da U niversidade Federal de Santa Catarina (U F SC), Bolsista da Coordenação de A perfeiçoamento de Pessoal de Nível Superior (CAPES), F lorianópolis, Santa Catarina, Brasil.

c D outora em Enfermagem, Professora Associada da Escola de Enfermagem da Universidade Federal do Rio Grande do Sul (UFRGS),

Bolsista de Produtividade em Pesquisa do Conselho N acional de Desenvolvimento Científico e T ecnológico (CN Pq), Porto Alegre, Rio

Grande do Sul, Brasil. 


\section{INT RODUÇÃO}

Os serviços hospitalares de emergência possuem características próprias que influenciam a organização do trabal ho e a gerência do cuidado. Eles estão inseridos no atual contexto político e estrutural do sistema de saúde brasileiro como 0 componente responsável pelo atendimento de situações graves em que há risco de morte e são necessárias intervenções rápidas e precisas. Porém, na prática, esses serviços também são utilizados por usuários com demandas que não se caracterizam clinicamente como urgências, de maneira a complementar a atenção recebida nas unidades básicas de saúde e unidades de pronto-atendimento, o que acar reta superlotação do serviço e sobrecarga de trabalho para os profissionais ${ }^{(1,2)}$.

O s enfer meiros que atuam nesses serviços são responsáveis, entre outras atividades, pela gerência do cuidado, que envolve o gerenciamento de recursos e a coor denação e articulação do trabal ho da equipe de enfer magem/ saúde, além da intermediação entre a família e a equipe de atendimento ${ }^{(3)}$. Compete a eles buscar meios para garantir a disponibilidade e qualidade de recursos materiais e de infra-estrutura que permitam à equipe atuar no atendimento às situações de urgência, visual izando as necessidades do paciente, conciliando os objetivos organizacionais e os da equipe de enfer magem, visando à produção de um cuidado integral e com maior qualidade $\mathrm{e}^{(4-6)}$.

0 processo de trabal ho do enfermeiro, como prática social integrante do trabalho coletivo em saúde, é composto por duas dimensões complementares: assistir e gerenciar. $\mathrm{N}$ a primeira, $\mathrm{o}$ enfermeiro tem como objeto de intervenção as necessidades de cuidado de enfermagem e por finalidade o cuidado integral; na segunda, o objeto de trabal ho é a organização do trabal ho e os recursos humanos em enfermagem, os meios e instrumentos são os diferentes saber es administrativos, materiais, equipamentos e instalações, além dos instrumentos técnicos da gerência, como: dimensionamento de pessoal, planejamento, educação continuada/ permanente, supervisão, avaliação de desempenho, os quais são empregados com a finalidade de criar e implementar condições adequadas à produção do cuidado e de desempenho da equipe de enfermagem ${ }^{(7-9)}$.

$\mathrm{N}$ essa linha de pensamento, considera-se a gerência como uma atividade meio para a atividade fim que é o cuidado, de modo que o gerenciamento do cuidado pode ser definido como a articulação entre a dimensão assistencial e a gerencial pa$\mathrm{ra}$ atender às necessidades de cuidado dos pacientes e os objetivos da instituição e da equipe de enfermagem ${ }^{(7-9)}$. 0 gerenciamento do cuidado implica tê-lo como foco das ações profissionais e utilizar os processos administrativos como tecnologias no sentido da sua concretização, por meio de ações diretas com os usuários ou por intermédio de delegação e articulação com outros profissionais da equipe de saúde. 0 enfermeiro gerencia o cuidado quando o planeja, o delega ou o faz, quando prevê e provê recursos, capacita a equipe de enfermagem e interage com outros profissionais, ocupando espaços de articulação e negociação em prol da consecução de melhorias do cuidado(7).

No entanto, a gerência do cuidado diante de um contexto com as especificidades apresentadas pel os ser viços de emergência, em que nem sempre há condições adequadas para realização de uma assistência qual ificada, não é uma tar efa fácil para os enfermeiros ${ }^{(10)}$, constituindo-se, portanto, uma problemática rel evante e pouco explorada na literatura científica da enfermagem ${ }^{(3)}$. A ssim, interroga-se: como os enfermeiros gerenciam o cuidado em um serviço hospitalar de emergência? Quais são as principais ações de gerência do cuidado realizada pelos enfermeiros nesses serviços?

Para buscar respostas a esses questionamentos, delineou-se este estudo com o objetivo analisar as ações de gerenciamento do cuidado realizadas pel os enfermeiros em um serviço hospitalar de emergência.

\section{MET ODOLOGIA}

T rata-se de um recorte de uma dissertação de M estrado ${ }^{(11)}$ que utilizou abordagem qual itativa do tipo estudo de caso. Esse tipo de estudo possibilita a investigação dos fenômenos no seu contexto real, analisando-os profunda e intensamente $\mathrm{e}^{(12)}$.

O cenário estudado foi o Serviço de Emergência de um Hospital Universitário localizado na região sul do Brasil, que atende pacientes nas especialidades clínica, cirúrgica, ginecologia e pediatria. Ele está organizado em cinco áreas de atendimento, conforme a complexidade do cuidado: A colhimento com Classificação de Risco, Sala de Internação Breve (SIB), Sala de Observação (SO) 
1 e 2, U nidade Vascular (UV) e Semi-intensiva (SI ) e U nidade Pediátrica (UP).

A coleta de dados ocorreu entre junho e setembro de 2009, a partir de observação participante e entrevista semi-estruturada com 20 enfermeiros do serviço de emergência mencionado. A seleção desses participantes ocorreu de forma intencional entre aquel es enfer meiros que aceitaram participar da pesquisa e trabalhavam há mais de seis meses no serviço de emergência. A definição desse período de tempo foi estabelecida com base na crença de que seis meses é um tempo suficiente à adaptação do profissional às rotinas do setor e à equipe de trabalho, podendo, desse modo, contribuir de for ma mais efetiva com a investigação.

Para realização das observações, utilizou-se um roteiro que focal izava o trabal ho ger encial dos enfermeiros e as relações entre os enfermeiros e integrantes da equipe de enfermagem e/ ou usuários durante o gerenciamento do cuidado. Foram realizados 40 períodos de observação durante todos os dias da semana e turnos de trabal ho, os quais tiveram duração variável de acordo com a qualidade da atenção despendida para a atividade, com média de duração em torno de 2 horas, perfazendo cerca de 90 horas de observação ao total. Para a realização das observações, utilizou-se um roteiro construído a partir do marco teórico-conceitual adotado no estudo e que estabelece como principais ações de gerência do cuidado: planejamento do cuidado, previsão e provisão dos recursos humanos e materiais, a coor denação do trabal ho, por meio da liderança e capacitação da equipe de enfermagem ${ }^{(8-10)}$. 0 registro das informações oriundas das observações foi realizado em um diário de campo.

As entrevistas contemplaram questões relacionadas às atividades dos enfermeiros no gerenciamento do cuidado no serviço de emergência. Realizaram-se 20 entrevistas, que foram gravadas em um dispositivo eletrônico de áudio, mediante autorização dos participantes da pesquisa, edepois transcritas. T anto o número de observações, quanto o de entrevistas real izadas foi estabelecido com base na saturação dos dados, ou seja, na repetição das informações, possibilitando a identificação de convergências e de um encadeamento entre as evidências ${ }^{(12)}$.

Para análise do material empírico, utilizou-se a técnica de análise de conteúdo temática ${ }^{(13)}$. N este artigo, são apresentados os resultados referen- tes à categoria temática denominada "A tuação dos enfermeiros no gerenciamento do cuidado".

O projeto foi aprovado pelo Comitê de Ética em Pesquisa da instituição de referência (parecer 09-151), foram seguidas as diretrizes e normas regulamentadoras de pesquisa envolvendo seres humanos e os participantes do estudo assinaram um T er mo de Consentimento Livre e E sclarecido em que concordavam em integrar a pesquisa. As observações e entrevistas foram codificadas, respectivamente, pela sigla OBS e letra E, associadas a números atribuídos confor me a realização de cada período de observação (OBS1, OBS2,..., OBS40) e entrevista (E 1, E 2,... E 20).

\section{RESULTADOS E DISCUSSÃO}

Para analisar a atuação dos enfermeiros no gerenciamento do cuidado em um serviço hospitalar de emergência, delimitaram-se três subcategorias que apresentam as principais ações real izadas pelos enfermeiros: planejamento do cuidado; previsão e provisão de recursos; e, supervisão, lider ança e capacitação da equipe de enfermagem.

\section{Planejamento do cuidado}

0 planejamento e a realização do cuidado contemplam a aplicação do processo de enfer magem e o controle sobre a real ização de exames laboratoriais e radiológicos.

A realização do processo de enfermagem possibilita aos enfermeiros a avaliação das condições de saúde dos pacientes e, desse modo, o planejamento e direcionamento das ações ter apêuticas que serão empreendidas em prol da sua recuper ação, bem como a del egação de atividades para equipe de enfermagem.

[...] o processo de enfermagem, mesmo com todas as coisas que tem para fazer, é um diferencial porque tu vai lá, olha o paciente, avalia, prescreve (E 2).

$\mathrm{N}$ a U P, colhe dados para realização do processo de enfer magem conver sando com 0 acompanhanteeas crianças sobre alimentação, sono e repouso, al terações re centes e outros aspectos conforme a situação de cada paciente $\mathrm{R}$ ealiza rápido examefísico, checa presença e estado dos acessos venosos (OBS16).

A execução do processo de enfermagem evidencia o papel do enfermeiro como gerente do cui- 
dado, pois permite a implementação de um plano de cuidados de enfer magem e uma aval iação constante da assistência. D esse modo, 0 enfer meiro pode favorecer a qual ificação e o mel hor direcionamento dos cuidados de enfer magem na busca de resultados específicos visando ao atendimento das necessidades do indivíduo.

De forma semel hante, para enfermeiros que atuam em um pronto-socorro, o processo de enfermagem configura-se como uma ferramenta gerencial que auxilia a planejar e organizar as ações da equipe de enfermagem ${ }^{(14,15)}$. 0 processo de enfermagem pode ser entendido como uma prática de gerenciamento do cuidado, na qual o profissional articula o seu fazer gerencial e assistencial para atender às necessidades de cuidado dos pacientes e ao mesmo tempo da equipe de enfermagem e da instituição(10).

Para avaliação e monitorização das condições de saúde dos pacientes, os exames laboratoriais e radiológicos são instrumentos diagnósticos amplamente utilizados nos ser viços hospitalares de emergência. A ssim, os enfermeiros estão sempre atentos aos exames realizados ou por realizar pelos pacientes, sendo essa uma atividade de apoio à equipe de saúde, principalmente para o profissional que os requisita.

[ ...] controledos exames, também éuma partegerencial. P rocuro ol har todos os exames dos pacientes, para ver se está faltando algo (E 11).

0 enfermeiro da SIB/SOI percebe que o funcionário responsável pel a real ização dos el etrocardiogramas está na unidade esolicita que el realize três exames em pacientes queaguardam pel o procedimento desdeo turno da tarde [ agora são 20h50min] (OBS23).

Os resultados dos exames laboratoriais e radiológicos dos pacientes na emergência são de suma importância para as decisões que envolvem, por exemplo, a al ta hospitalar de um paciente ou a indicação de que ele inspira maiores cuidados e uma monitorização mais contínua. I sso explica a preocupação dos enfermeiros em controlar a realização dos exames diagnósticos prescritos aos pacientes no serviço de emergência.

O planejamento do cuidado contempla não só a relação dos enfermeiros com os usuários, mas também com os familiares dos pacientes/ acompanhantes dos pacientes, pois cabe a esses profissionais, via de regra, decidir quanto à entrada e perma- nência deles no serviço de emergência. Entretanto, a presença dos familiares dos pacientes no serviço de emergência é uma questão controversa entre os enfermeiros.

[ ...] a gentesabequeéimportante para o paciente. $M$ as daqui a pouco tem uma urgência e tem que mandar tirar. E aí o familiar não gosta, o paciente não gosta [...] (E1).

[ ...] tenho muita dificuldade de lidar com os familiares, les ficam pressionando, exigindo procedimentos que nós e os funcionários não conseguimos faz er porquenão tem tempo [ ...] (E 15).

Os enfermeiros reconhecem que a visita dos familiares é importante para a recuperação dos pacientes no serviço de emergência, porém diante de al gumas situações, como 0 atendimento a uma urgência, a presença deles pode se tornar um empeciIho à realização do trabal ho, em função, por exemplo, da atenção que requerem e/ ou dos questionamentos que realizam. Essa dificuldade pode ser explicada pelos resultados de um estudo em que a orientação e 0 atendimento às necessidades dos familiares foram identificados como duas das atividades mais estressantes dos enfermeiros de pronto socorro dos hospitais brasileiros ${ }^{(16)}$. A superação desse problema perpassa a adoção de estratégias pelos serviços de emergência e de novas posturas dos enfer meiros para 0 acol himento dos familiares dos pacientes e suas demandas.

\section{Previsão e provisão de recursos}

As principais ações de gerência do cuidado dos enfermeiros visando à previsão e provisão de recursos para produção do cuidado foram: elaborar a escala mensal de funcionários, realizar a distribuição diária dos funcionários e gerenciar recursos materiais.

A elaboração da escala mensal e distribuição diária dos funcionários apareceram com relevância entre as atividades gerenciais dos enfermeiros. Considerando a dinamicidade do trabal ho em emergência, a distribuição diária dos funcionários entre os setores da unidade está sempre sujeita a modificações, pois os técnicos de enfer magem podem ser deslocados de uma área para outra conforme 0 aumento ou diminuição do número de pacientes ao longo de um turno de trabalho. 
D esde 0 momento da chegada, verificar escala e ver quantos pacientes tem em cada sala, para que não fique um número de técnicos a mais ou a menos do que necessita, e daí tu podes deslocar isso e jogar as pessoas de uma sala para outra para poder trabalhar melhor (E 14).

A pós a passagem de plantão, 0 enfermeiro da U P diz aos demais enfermei ros: "E stou com a pediatria lotada, tem consulta agendada atéa meia-noitee só tenho uma funcionária". E nfermeiro S01: "E u também, já viu como está a SIB ?". A enfer meira da U V, então, conversa com uma das técni cas de enfer magem da tardee pede se ela não poderia ficar à noite também (OBS22).

A correta elaboração das escalas e distribuição dos funcionários garante que em cada setor da emergência tenha um número suficiente de trabaIhadores de enfer magem, conforme o quantitativo de pessoal disponível. Para tanto, avaliar e trocar infor mações constantemente sobre o ritmo de trabalho nos diferentes setores da emergência, para efetuar trocas conforme as mudanças e novas demandas vão surgindo ao longo do turno. T ais achados articulam-se aos resultados de outros estudos em que a elaboração de escalas de trabal ho e cobertura de turnos com déficit de pessoal foram identificadas como atividades gerenciais fundamentais na organização e divisão do trabalho, principal mente diante da instabilidade que envolve a rotina e a demanda de atendimentos em emergên$\mathrm{cia}^{(10,14,15)}$

0 gerenciamento de recursos materiais foi expresso pelas atividades dos enfer meiros relacionadas ao controle da quantidade e qualidade dos materiais e equipamentos e é fundamental para garantir a qualidade da assistência. Os enfermeiros atuam na recepção, no armazenamento, distribuição e controle dos materiais na unidade.

R evisando o box de urgência, tem que estar tudo pronto, não tem como correr para farmácia ou buscar um material quando está atendendo paciente (E 4).

[ ...] após a troca de plantão, existem tarefas para fazer: verificar controlados e geladeira do sangue, revisar carro de parada, verificar se o box de urgência está completo ese tem material para trabalhar (E 14).

0 gerenciamento de recursos materiais envolve 0 fluxo de atividades de programação, compra, recepção, armazenamento, distribuição e con- trole, com o objetivo de garantir que a assistência aos usuários não sofra inter rupções por insuficiência na quantidade ou na qualidade de materiais ${ }^{(17)}$. A previsão da quantidade de material gasto pela unidade e uma análise para verificar a suficiência dos materiais é uma das ações gerenciais descritas em um estudo sobre o papel do enfermeiro de uma U nidade de Terapia Intensiva no gerenciamento de recursos materiais(18).

No concer nente à estrutura física do serviço de emergência, o número de macas disponíveis preocupa os enfermeiros e, muitas vezes, dificulta a gerência do cuidado. Diante da falta de leitos para acomodar todos os pacientes, é comum os enfermeiros solicitarem macas extras às unidades de inter nação para deitar os pacientes mais debilitados e/ ou acomodá-los nos consultórios para que possam descansar um pouco à noite.

[ ...] superlotou? precisa de mais macas? a gente pede emprestado (E 1).

Ver os pacientes com mais restrição e tentar uma maca, derepente colocar um clienteno consultório à noitepara promover um pouco mais de confor to (E 7).

[ ...] tu não consegues imaginar o estressequeéver um paciente hipotenso, mal e tu não ter maca para colocar ele, tu ter que levantar um para poder deitar outro (E 15).

M édico solicita ao enfermeiro responsável pela SIB/ SO 1 que ele consiga uma maca para uma senhora idosa em uma cadeira de rodas (OBS6).

A falta de macas para todos os pacientes do serviço de emergência confere aos enfermeiros a difícil e estressante atribuição de decidir quais pacientes serão acomodados nelas. Os médicos, com frequência, solicitam que os enfer meiros providenciem macas para os pacientes mais g raves e/ ou debilitados deitarem. Para isso, el es procuram solicitar macas emprestadas ou transferir pacientes de macas para cadeiras e vice-versa. T ais ações têm caráter paliativo e, muitas vezes, são fonte de sofrimento no trabal ho. D essa forma, é importante que os enfermeiros se mobilizem e discutam as questões relacionadas à organização e estruturação dos serviços de emergência, pois a responsabilidade pela provisão e previsão dos recursos e da infra-estrutura necessários ao cuidado não é uma responsabilidade exclusiva da enfer magem. 


\section{Supervisão, liderança e capacitação da equipe de enfermagem}

A super visão, liderança e capacitação da equipe de enfermagem integram um importante eixo do gerenciamento do cuidado, tendo em vista a multiplicidade de atividades desenvolvidas pelos técnicos de enfermagem sob coordenação dos enfermeiros.

[ ...] nós somos a chefia deles no turno, tem que ver se eles estão faz endo as suas funções cor retamente, se eles não estão ficando muito tempo no intervalo ou alguma coisa que eles não perceberam no paciente (E3).

P rincipalmente, coordenar o que os técnicos devem ou não fazer. M uitas vez es, eles estão com muitos pacientes e não tem noção do que é prioridade, então tem que dizer o que tem que ver primeiro (E 16).

A principal preocupação dos enfermeiros ése os técnicos de enfermagem estão cumprindo corretamente com suas atividades e conseguindo elencar aquelas que são prioritárias diante da grande demanda de trabal ho no serviço de emergência. E ssa preocupação remete à utilização da supervisão como um instrumento do processo de trabalho gerencial do enfermeiro. Entendida como responsável por promover a reflexão e discussão sobre a execução da prática com base no acompanhamento do cotidiano do trabal ho, a supervisão, no entanto, é lembrada pelos enfermeiros, principalmente, na sua dimensão de controle, que se direciona ora para o trabalhador ora para o processo de trabalho, na verificação do que foi realizado. Resultados semelhantes estão descritos em uma pesquisa sobre o trabalho gerencial de enfermeiros no contexto hospitalar ${ }^{(10)}$.

A liderança é um dos principais instrumentos do enfermeiro para a gerência dos processos de trabal ho, coordenação e articulação das atividades que envolvem a produção do cuidado em saúde e enfermagem, bem como dos profissionais que as desempenham.

[ ...] tu conseguir ser líder da tua equipe, que a tua equipe te veja como um líder [ ...] (E2).

[ ...] é um ponto chave o enfer meiro ser líder e aprender a liderar e comandar toda a unidade, porque ele tem como papel ser 0 integrador de todas as equipes (E8).

A liderança auxilia o enfermeiro no gerenciamento do cuidado, favorecendo o planejamento da assistência, a coordenação da equipe de enfermagem, a del egação e distribuição de atividades. Ela também é importante para o bom funcionamento da unidade, tendo em vista a centralidade dos enfermeiros e o papel articulador e integrador que é exercido por eles na emergência. D e forma semeIhante, enfer meiros de um pronto socorro também consider am a liderança uma competência gerencial essencial em decorrência da dinâmica do trabalho em emergência(15).

A realização de capacitações com a equipe de enfermagem foi citada pelos depoentes como uma estratégia para melhorar 0 atendimento aos pacientes no serviço de emergência.

[ ...] tem toda a questão gerencial com os técnicos de enfermagem, dos treinamentos (E 2).

[ ...] promover ações de ensino, de qualificação para a equipetécnica poder prestar um atendimento mais ade quada para o cliente (E 7).

A realização de treinamentos e ações de ensino com os técnicos de enfer magem são instrumentos gerenciais utilizados pelos enfermeiros para qualificar o cuidado prestado pela equipe de enfermagem no serviço de emergência. Resultado similar está descrito em uma pesquisa sobre a assistência de enfermagem na sala de emergência de uma U nidade de A tendimento Pediátrico(5).

A pesar da importância das atividades de qualificação profissional por meio de programas de educação continuada, vale mencionar que são crescentes as discussões em torno da importância da adoção de práticas de educação per manente na área da saúde. A educação per manente baseia-se no pressuposto de que o processo de trabal ho é o gerador das necessidades de conhecimento e das demandas educativas contínuas e utiliza a metodologia da problematização e a aprendizagem significativa nos processos educativos de trabalhadores de saúde para a melhoria da qualidade dos serviços e a transformação das práticas de saúde e enfermagem ${ }^{(19-21)}$ A ssim, pontua-se a necessidade de que as práticas educativas dos enfermeiros com a equipe de en- 
fermagem sejam pensadas e realizadas a partir da perspectiva da educação permanente.

\section{CONSIDERAÇÕES FINAIS}

Ao analisar as ações dos enfermeiros no gerenciamento do cuidado em um serviço de emergência, este estudo possibilitou a visualização e discussão de al gumas possibilidades de articulação entre as dimensões gerencial e assistencial do trabalho do enfermeiro, por meio do planejamento do cuidado, da previsão e provisão de recursos e da supervisão, liderança e capacitação da equipe de enfermagem.

A atuação dos enfermeiros no planejamento do cuidado foi evidenciada por meio da aplicação do processo de enfermagem e do controle sobre a realização dos exames labor atoriais e radiológicos. As principais práticas gerenciais dos enfermeiros visando à previsão e provisão de recursos para a produção do cuidado em no serviço de emergência foram: elaboração da escala mensal de funcionários, realização da distribuição diária dos funcionários e gerenciamento dos recursos materiais. Por meio das atividades de supervisão, lider ança e capacitação da equipe de enfermagem, os enfermeiros coordenam a realização do cuidado e zelam pela qualidade da assistência prestada.

Os resultados podem fomentar a discussão e reflexão de enfermeiros sobre suas práticas, contribuindo com a compreensão da importância do gerenciamento do cuidado realizada pelos enfermeiros na mel horia da assistência e das práticas de atenção à saúde nos serviços de emergência. N esse sentido, algumas sugestões e recomendações são possíveis a partir dos achados da pesquisa. Q uanto à presença dos familiares dos pacientes no serviço de emergência, é importante sensibilizar os profissionais da equipe de enfermagem, em especial os enfermeiros, para inclusão da família no seu projeto ter apêutico, pois a interação e comunicação com a equipe de atendimento podem amenizar a dor, 0 estresse e o sofrimento dos familiares. A supervisão como um instrumento gerencial deve ser utilizada pelos enfermeiros como um dispositivo na busca da emancipação e desenvolvimento da cidadania dos agentes do processo de trabalho.

\section{REFERÊNCIAS}

1 Dal Pai D, Lautert L. 0 trabal ho em urgência e emergência e a relação com a saúde das profissionais de enfermagem. Rev Latino-Am Enfermagem. 2008; 16(3):439-44.

2 G arlet ER, Lima M ADS, Santos J LG, M arques G Q. $F$ inalidade do trabalho em urgências e emergências. Rev Latino-Am Enfermagem. 2009;17(4):535-40.

3 Santos J L G, G ehlen GC, W eis A H, G arlet ER, Lima MADS. Nursing practice in emergency care: systematic review. Online Braz J N urs [ Internet] . 2009 [ cited 2009 D ec 20] ;8(3). Available from: http:/ / www.uff.br/ objnursing/ index.php/ nursing/ article/ view/ 1243

4 W ehbe G, Galvão CM . 0 enfermeiro de unidade de emergência de hospital privado: algumas considerações. Rev Latino-Am Enfermagem. 2001;9(2):8690.

5 T acsi Y RC, Vendruscolo D M S. A assistência de enfermagem no serviço de emergência pediátrica. Rev Latino-A m Enfermagem. 2004;12(3):477-84.

6 Lima SBS, Erdmann AL. A enfermagem no processo da acreditação hospitalar em um serviço de urgência e emergência. Acta Paul Enferm. 2006;19(3):271-8.

7 Rossi FR, Silva M AD. Fundamentos para processos gerenciais na prática do cuidado. Rev E sc Enferm USP. 2005;39(4):460-8.

8 F elli VEA, Peduzzi M . 0 trabal ho gerencial em enfermagem. I n: K urcgant $P$, organizadora. G erenciamento em enfermagem. R io de Janeiro: G uanabara Koogan; 2005. p. 1-13.

9 Hausmann M , Peduzzi M. Articulação entre as dimensões gerencial e assistencial do processo de trabal ho do enfermeiro. Texto Contexto Enferm. 2009; 18(2):258-65.

10 Peixoto M GS. A bordagem gerencial do cuidado de enfermagem em emergência [ dissertação] . Rio de Janeiro: E scola de E nfermagem, U niver sidade F ederal do Rio de Janeiro; 2004.

11 Santos J LG. A dimensão gerencial do trabal ho do enfermeiro em um serviço hospitalar de emergência [ dissertação] . Porto A legre: E scola de E nfer magem, U niversidade Federal do Rio G rande do Sul; 2010.

12 Y in RK . Estudo de caso: planejamento e métodos. 3 a ed. Porto A legre: Bookman; 2005.

13 Bardin L. A nálise de conteúdo. Lisboa: E dições 70; 2011. 
14 Y wata G CC, Danski M T R, M ingorance P, Pedrolo E, L azzari L SM . A prática do enfer meiro assistencial em um serviço de pronto atendimento adulto. Cogitare Enferm. 2009;14(4):734-9.

15 M ontezeli J H. O trabalho do enfermeiro no prontosocorro: uma análise na perspectiva das competências gerenciais [ dissertação]. Curitiba: Programa de Pós-G raduação em E nfer magem, U niver sidade Federal do Paraná; 2009.

16 M enzani G, Bianchi ERF. Stress dos enfer meiros de pronto socorro dos hospitais brasileiros. Rev Eletrônica Enferm [ Internet] . 2009 [ citado 2010 fev 10];11(2):327-33. Disponível em: http:/ / www.fen. ufg.br/ revista/ v11/ n2/ v11n2a13.htm.

17 Castilho V, G onçalves VL M. G erenciamento de recursos materiais. In: Kurcgant P, organizadora. G erenciamento em enfermagem. São Paulo: Guanabara Koogan; 2005. p. 157-70.
18 O liveira N C, Chaves LD P. G erenciamento de recursos materiais: o papel do enfermeiro de UTI. Rev RENE. 2009;10(4):19-27.

19 M erhy EE, Feurwerker LCM , Ceccim RB. E ducación per manente en salud: una estrategia para intervir en la micropolítica del trabajo en salud. Salud Colect. 2006;2(2):147-60.

20 Peduzzi M , D el Guerra D, Braga CP, L ucena F S, Silva JAM. A tividades educativas de trabal hadores na atenção primária: concepções de educação per manente e educação continuada em saúde presentes no cotidiano de unidades básicas de saúde em São PauIo. Interface Comun Saúde E duc. 2009;13(30):12134.

21 A mestoy SC, Schveitzer M C, M eirelles BH S, Backes VM S, E rdmann A L. Paral el o entre educação per manente em saúde e administração complexa. Rev G aúcha Enferm. 2010;31(2):383-7.

\author{
Endereço do autor / Dirección del autor / \\ Author's address: \\ José L uís $\mathrm{G}$ uedes dos Santos \\ Programa de Pós-G raduação em E nfer magem daU F SC \\ Centro de Ciências da Saúde \\ Campus U niversitário, s/ n-, Bairro T rindade \\ 88040-970, F lorianópolis, SC \\ E-mail: joseenfermagem@gmail.com
}

Recebido em: 05/ 04/ 2011

A provado em: 08/ 09/2011 